\title{
Flow and Turbulence driven Water Surface Roughness and Gas Exchange Velocity in Streams
}

\author{
Christian Noss ${ }^{1, *}$, Pascal Bodmer ${ }^{1}$, Kaan Koca ${ }^{1}$, and Andreas Lorke $^{1}$ \\ ${ }^{1}$ Institute for Environmental Sciences, University of Koblenz-Landau, Landau, Germany
}

\begin{abstract}
Gas exchange velocity in streams and rivers controls fluxes of atmospheric gases across the air-water interface and is commonly related to the turbulence at the water side. Similarly, river flow hydraulics influences the water surface roughness, which is frequently used (in terms of surface flow types) for eco- and morphological mapping of spatial variations of hydraulic conditions. We investigated the relationships between gas exchange velocity, water surface roughness and flow hydraulics for different surface flow types in a low-mountain stream. We used the flux chamber-method to estimate exchange velocity, a freely floating sphere (equipped with acceleration sensors) to measure water surface roughness, as well as a field-particle image velocimetry system for flow and turbulence measurements. The results demonstrate that the gas exchange velocity in smooth and rippled flows followed the same universal dependence on turbulent dissipation rates (with an empirical scaling coefficient at the upper limit) as observed in wind-driven systems. More rough flows were anisotropic and gas exchange velocity was stronger related to vertical components of turbulence parameters. We further explored the potential of using surface flow type evaluations and water surface roughness measurements for estimating gas exchange velocities at the reach scale and beyond.
\end{abstract}

\section{Introduction}

Estimations of gas fluxes across the air-water interface of streams are of particular importance for the determination of reaeration processes or fluxes of greenhouse gases [1, 2 ]. The diffusive flux of atmospheric gases is the product of the difference of dissolved gas concentration in the stream and in the atmosphere and the gas exchange velocity $(k)$. The gas exchange velocity is mainly controlled by the turbulent mixing on the water side [3]. Physically-based models relate the gas exchange velocity to the near-surface turbulence where the most efficient eddy size, transporting gases near the interface, scales with the Kolmogorov microscale, leading to [4]:

$$
k=\alpha(\varepsilon v)^{1 / 4} S c^{-1 / 2} \text {. }
$$

* Corresponding author: noss@uni-landau.de 
$\alpha$ denotes a scaling factor, $\varepsilon$ the dissipation rate of turbulent kinetic energy, $v$ the kinematic viscosity of water, and $S c$ the Schmidt number, which is defined by the ratio between the kinematic viscosity of water and the molecular diffusivity of the gas. Several field and lab studies (e.g., conducted in lakes and open channel flows [1, 5]) support Eq.1. However, detailed simultaneous in-situ surveys of dissipation rate and gas exchange velocity are difficult, and to our knowledge no study to date validates Eq.1 for streams and gravity driven turbulent flows based on field measurements.

In contrast to physically-based models, the variability of gas exchange velocity (or aeration coefficient, which also includes water depth) observed in streams has been empirically related to bulk hydrometric data $[2,6,7]$. These relationships account for the flow and turbulence driven gas exchange velocities through measured or estimated slope, velocity and depth, and enable large-scale assessments. As an alternative or addition to such methods, we suggest to use visual surveys of different surface flow types [8] as a proxy, which might be suitable for characterizing gas exchange velocities in stream sections.

Here we investigate the physically-based relationship of Eq.1 by in-situ measurements of dissipation rates and gas exchange velocities at different sections (distinguished by their predominant surface flow type) in a stream and compare them with universal dependences observed in flumes and in wind-driven systems. We further investigate the relationships between gas exchange velocity, bulk hydrometrics and water surface roughness of the sections. A new method is presented, which enables an empirical but quantitative estimation of the water surface roughness. Finally, we show a close agreement of the results with the qualitative assessment of surface flow type evaluation.

\section{Methods}

\subsection{Study Site}

Gas exchange velocities, water surface roughness and turbulence were measured at four sections in the Wellbach, a small, fine-substrate dominated, siliceous highland stream [9]. The Wellbach is a third order (Strahler) headwater stream located in the Palatinate-Forest in Southwest Germany. Four different sections (Fig.1a-d) with distinct flow conditions in respect to different surface flow types (SFT) were selected and visually assessed following the categorization of Newson and Newson [8]: smooth boundary turbulent (SBT), rippled flow (RIP), unbroken standing wave (USW) and broken standing wave (BSW).

The flow of the SBT section with only small turbulent cells disturbing the smooth water surface can be denoted as a glide flow. The RIP section with continuous downstream propagating ripples on the water surface corresponds to a run flow. The USW section is a riffle flow, where standing waves appear above the steep and coarse gravel and cobble riverbed. BSW are associated with local cascade flows, i.e. chute and surging flows above and downstream of protruding boulders. SBT, RIP and USW are related to quasihomogeneous riverbed and flow characteristics of corresponding approximately $15 \mathrm{~m}$ long sections (Table 1). In contrast, BSW do not extent across the entire stream width, hence is not related to the bulk hydrometric of the corresponding section.

Water surface roughness was measured along the entire section lengths, while gas exchange velocities and turbulence were measured at two to four sampling spots of a characteristic SFT section (except BSW, where only surface roughness of spill and hydraulic jump was measured and turbulence measurements were impossible, see Section 2.2.). All measurements were conducted between $2^{\text {nd }}$ and $15^{\text {th }}$ December 2016 . 

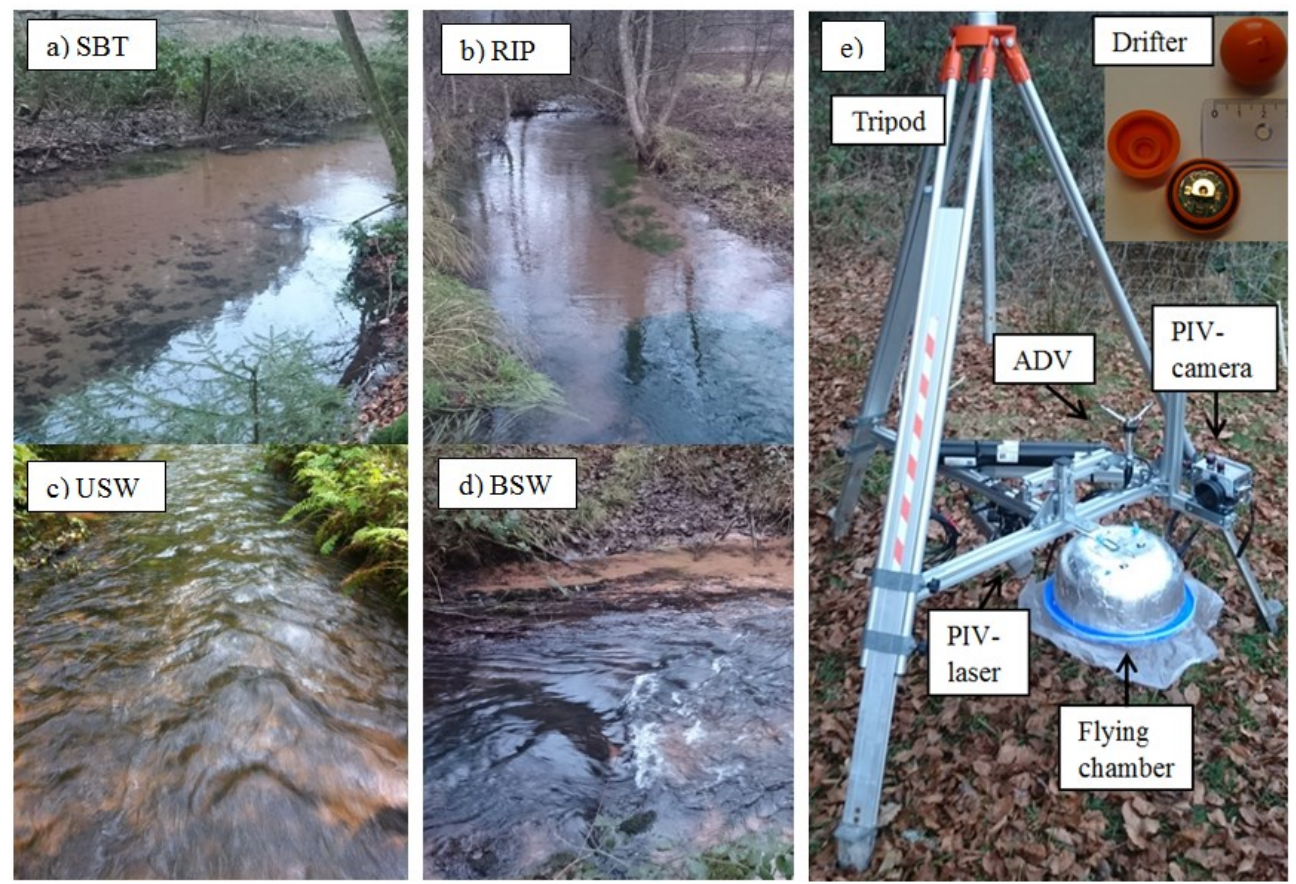

Fig. 1. Study sites at the Wellbach, a) smooth boundary turbulent (SBT), b) rippled flow (RIP), c) unbroken standing wave (USW), and d) broken standing wave (BSW), and instrumentation (e) for gas exchange velocity and turbulence (tripod), and water surface roughness (inset picture: drifter) measurements.

\subsection{Setup and Sampling}

At each spot, measurements of gas exchange velocities and turbulence were conducted with the flying chamber and PIV-system attached to a tripod (Fig.1e). The tripod, hence also the longitudinal axis of the PIV-laser below the centerline of the chamber, was aligned against the main flow direction (vertical laser light sheet).

Gas exchange velocities at the spots of SFT sections were obtained with a three-step procedure. First, fluxes of carbon dioxide $\left(F_{\mathrm{CO} 2}\right)$ across the sediment water interface were measured from the linear increase of $\mathrm{CO}_{2}$ partial pressure (over periods ranging between 24 $\mathrm{s}$ to $2.3 \mathrm{~min}$.) in the headspace of a flying chamber [10]. The flying chamber was connected to a Greenhouse Gas Analyzer (UGGA; Los Gatos Research, Inc., USA) via two 4.65meter-long gas-tight tubes. Second, partial pressures of $\mathrm{CO}_{2}\left(p \mathrm{CO}_{2}\right)$ in air (directly) and water samples were measured on-site using the UGGA. For the water samples, we applied the headspace method (one sample before, during and after each spot measurement) conducted with closed-loop as described in Alshboul et al. [11]. Third, the gas exchange velocity for $\mathrm{CO}_{2}$ was then calculated from

$$
k=F_{\mathrm{CO} 2} /\left(K_{\mathrm{H}} \Delta p \mathrm{CO}_{2}\right),
$$

where $K_{\mathrm{H}}$ denotes the Henry's constant adjusted for in-situ water temperature [12] and $\Delta p \mathrm{CO}_{2}$ is the difference of the partial pressure of $\mathrm{CO}_{2}$ in water and in the atmosphere. The gas exchange velocity of $\mathrm{CO}_{2}$ at in-situ temperature was further standardized to $k_{600}$; i.e., $k$ for a Schmidt number $(S c)$ of 600 [3]:

$$
k_{600}=k(S c / 600)^{1 / 2} .
$$


Table 1: Bulk hydrometrics of smooth boundary turbulent (SBT), rippled flow (RIP) and unbroken standing wave (USW) sections: mean flow depth $(H)$, mean stream width $(W)$, water surface slope $(S)$, mean flow speed ( $U_{S}$, arithmetic mean over 50-55 measurements), Froude number $(F r)$, Reynolds number $(R e)$ and energy dissipation rates estimated from uniform channel drag ( $\varepsilon_{D}$, Eq. 7$)$ and from bed shear stress $\left(\varepsilon_{S}\right.$, Eq. 6).

\begin{tabular}{ccccccccc}
$\begin{array}{c}\text { Surface } \\
\text { flow type }\end{array}$ & $\begin{array}{c}H \\
(\mathrm{~m})\end{array}$ & $\begin{array}{c}W \\
(\mathrm{~m})\end{array}$ & $\begin{array}{c}S \\
(\%)\end{array}$ & $\begin{array}{c}U_{S} \\
\left(\mathrm{~m} \mathrm{~s}^{-1}\right)\end{array}$ & $\begin{array}{c}F r \\
(-)\end{array}$ & $\begin{array}{c}R e \\
(-)\end{array}$ & $\begin{array}{c}\varepsilon_{D} \\
\left(\mathrm{~W} \mathrm{~kg}^{-1}\right)\end{array}$ & $\begin{array}{c}\varepsilon_{S} \\
\left(\mathrm{~W} \mathrm{~kg}^{-1}\right)\end{array}$ \\
\hline SBT & 0.31 & 5.22 & 0.02 & 0.17 & 0.10 & $3.1 \cdot 10^{4}$ & $3.3 \cdot 10^{-4}$ & $4.1 \cdot 10^{-5}$ \\
RIP & 0.40 & 3.59 & 0.04 & 0.30 & 0.15 & $6.5 \cdot 10^{4}$ & $1.2 \cdot 10^{-3}$ & $1.1 \cdot 10^{-4}$ \\
USW & 0.23 & 3.24 & 1.06 & 0.76 & 0.51 & $1.0 \cdot 10^{5}$ & $7.9 \cdot 10^{-2}$ & $1.3 \cdot 10^{-2}$
\end{tabular}

The water surface roughness was measured by a small $(28 \mathrm{~mm}$ diameter) spherical shaped drifter. This drifter was equipped with a 9-axis orientation sensor (Bosch BNO55), electronical periphery and batteries. Only raw acceleration data sampled at $50 \mathrm{~Hz}$ and with $0.0024 \mathrm{~m} \mathrm{~s}^{-2}$ resolution were used for further analyses, because drifter orientation, which could be estimated by the sensor, failed in the present drifter application. Although readings are affected by the (constant) gravitational acceleration, standard deviation of the norm of accelerations $\left(S D_{\mathrm{a}}\right)$ enables one to quantify water surface roughness, more precisely buoyancy-driven vertical oscillations of the drifter at the water surface and predominantly horizontal velocity gradients, which are related to the water surface roughness. Multiple drifter runs (SBT: 13 runs, RIP: 28 runs, USW: 10 runs, BSW: 16 runs) were conducted and averaged for water surface roughness estimations.

Turbulence measurements were conducted by a custom-made particle image velocimetry (PIV) system, which consists of a digital camera (Sony RX100IV) and a green continuous wave laser (Hercules-225, Laserglow) with a Powell-lens to expand the laser beam to a planar light sheet with an opening angle of $60^{\circ}$. Illuminated natural particles were recorded at $120 \mathrm{~Hz}$ in a $416 \mathrm{~mm}$ wide and $224 \mathrm{~mm}$ high field of view of the camera (top end $20 \mathrm{~mm}$ below the water surface) for at least $3 \mathrm{~min}$. Instantaneous two-dimensional velocity vectors at a spatial resolution of $8 \mathrm{~mm} \times 8 \mathrm{~mm}$ were calculated using a three-step adaptive correlation algorithm with final interrogation areas of $64 \times 64$ pixel and $50 \%$ overlap with the software DynamicStudio V (Dantec Dynamics). In the adaptive correlation procedure initial velocity vectors are estimated in large interrogation areas $(256 \times 256$ pixel) and subsequently refined in smaller sized interrogation areas. Spurious velocity vectors were identified by peak validation and replaced by a moving average spanning $7 \times 7$ interrogation areas.

Reynolds-decomposition was applied to the velocity time series to obtain mean and instantaneous velocity fluctuations of the longitudinal $\left(\bar{u}, u^{\prime}\right)$ and vertical $\left(\bar{w}, w^{\prime}\right)$ component. Power-spectral density $\left(P_{u}, P_{w}\right)$ of velocity fluctuations was estimated from unfiltered velocity time series using Welch's method [13]. Frequency spectra were converted to wavenumber spectra using Taylor's hypothesis of frozen turbulence $(\kappa=f / \bar{u}$, frequencies $(f)$ and wavenumbers $(\kappa)$ are reported as cycles per second and per meter, respectively). Spectral analysis revealed that noise dominated $f>10 \mathrm{~Hz}$. Hence, a zerophase low-pass filter $\left(12^{\text {th }}\right.$ order Butterworth) with corresponding cut-off frequencies was applied to the velocity time series, after de-spiking using the method described in Goring and Nikora [14]. Turbulent kinetic energy was estimated from the filtered velocities:

$$
T K E=0.5\left(\overline{u^{\prime 2}}+2 \overline{w^{\prime 2}}\right)
$$


Dissipation rates of turbulent kinetic energy $\left(\varepsilon_{u}, \varepsilon_{w}\right)$ were estimated using the inertial dissipation technique [15]. A constant slope of $\kappa^{-5 / 3}$ was fitted to the inertial subrange in measured wavenumber $\left(\kappa^{-}\right)$spectra to match the theoretical spectrum:

$$
P(\kappa)=\bar{u} P(f / \bar{u})=2 \pi A \varepsilon^{2 / 3}(2 \pi \kappa)^{-5 / 3},
$$

where $A=0.49$ for $P_{u}$ and $A=0.65$ for $P_{w}$ [15]. Turbulence measurements were impossible at BSW spots due to the shallow water depth, large boulder and air bubble entrainment at the hydraulic jump (Fig.1d).

Additionally, section scale surveys of water level slope $(S)$, stream width $(W)$ and flow depth $(H)$, as well as near surface flow velocities $\left(U_{\mathrm{S}}\right)$ were conducted with a surveyor's level and an electro-magnetic current meter (OTT MF Pro). These enable to estimate Reynolds and Froude number as well as the section scale dissipation rate by assuming a boundary-layer flow, where energy dissipation is governed by bed shear stress (indirectly) $[1]$,

$$
\varepsilon_{S}=\left(g R_{h} S\right)^{3 / 2} / H
$$

where $g$ denotes gravitational acceleration, and on the assumption that channel drag and corresponding energy dissipation are uniformly distributed over depth

$$
\varepsilon_{D}=g S U_{S} .
$$

\section{Results}

\subsection{Gas Exchange Velocity and Fluxes}

The gas exchange velocity at the study sites increased with the surface flow type from the smoothest, SBT, to the roughest, BSW (Table 2). Measured partial pressures of dissolved $\mathrm{CO}_{2}$ in the stream water were in all samplings higher than the partial pressure in the atmosphere (494 ppm); hence, the stream emitted $\mathrm{CO}_{2}$ to the atmosphere. Because partial pressures of dissolved $\mathrm{CO}_{2}$ were similar at all sites and during the measurement period, fluxes increased - similar to the gas exchange velocity - with the surface flow type, from SBT to BSW.

Table 2: Mean values ( \pm standard deviation) of fluxes, gas exchange velocities $\left(k_{600}\right)$, and partial pressure $\left(p \mathrm{CO}_{2}\right)$ of dissolved $\mathrm{CO}_{2}$, as well as corresponding flow (mean velocity $U$ ) and turbulence (turbulent kinetic energy TKE, dissipation rate estimated from longitudinal $\varepsilon_{u}$ and vertical velocities $\varepsilon_{w}$ ) parameter from spot surveys at the investigated sections.

\begin{tabular}{cccccccc}
$\begin{array}{c}\text { Spots } \\
\text { at }\end{array}$ & $\begin{array}{c}\text { Flux } \\
(\mathrm{mmol} \\
\left.\mathrm{m}^{-2} \mathrm{~d}^{-1}\right)\end{array}$ & $\begin{array}{c}k_{600} \\
\left(\mathrm{~m} \mathrm{~d}^{-1}\right)\end{array}$ & $\begin{array}{c}p \mathrm{CO}_{2} \\
(\mathrm{ppm})\end{array}$ & $\begin{array}{c}U \\
\left(\mathrm{~m} \mathrm{~s}^{-1}\right)\end{array}$ & $\begin{array}{c}T K E \\
\left(10^{-3} \mathrm{~J}\right. \\
\left.\mathrm{kg}^{-1}\right)\end{array}$ & $\begin{array}{c}\mathcal{E}_{\mathrm{u}} \\
\left(10^{-3} \mathrm{~W}\right. \\
\left.\mathrm{kg}^{-1}\right)\end{array}$ & $\begin{array}{c}\mathcal{E}_{\mathrm{W}} \\
\left(10^{-3} \mathrm{~W}\right. \\
\left.\mathrm{kg}^{-1}\right)\end{array}$ \\
\hline SBT & $0.10 \pm 0.01$ & $7.19 \pm 0.45$ & $817 \pm 9$ & $0.38 \pm 0.03$ & $1.8 \pm 0.5$ & $0.16 \pm 0.05$ & $0.13 \pm 0.04$ \\
RIP & $0.13 \pm 0.02$ & $9.63 \pm 1.44$ & $811 \pm 5$ & $0.52 \pm 0.02$ & $5.2 \pm 3.0$ & $0.68 \pm 0.53$ & $0.48 \pm 0.30$ \\
USW & $0.21 \pm 0.04$ & $12.82 \pm 2.54$ & $887 \pm 34$ & $1.04 \pm 0.31$ & $18 \pm 7.1$ & $25.0 \pm 24.0$ & $4.1 \pm 2.7$ \\
BSW & $0.27 \pm 0.05$ & $26.38 \pm 5.23$ & $742 \pm 1$ & -- & -- & -- & --
\end{tabular}

\subsection{Water Surface Roughness}

The standard deviations of acceleration of multiple runs at the study sites reflect the visual impression of different surface roughness in terms of propagating or standing waves with 
different sizes. Largest values were obtained at the BSW section followed by the USW (Fig.2a, Fig.1c, d). There the water surface was much rougher in comparison to that of RIP and SBT (Fig.1a, b). Mean travel times ( \pm standard deviation) of the Lagrangian drifter were $40.0 \pm 9.6 \mathrm{~s}$ at SBT, $35.2 \pm 15.6 \mathrm{~s}$ at RIP, and $14.4 \pm 0.9 \mathrm{~s}$ at USW. Sequences, where the drifter passed the BSW (i.e., the hydraulic jump from drifter immerging until leaving the broken standing wave) were shorter $(5.1 \pm 1.6 \mathrm{~s})$. The drifter took different pathways, i.e. it passed different subregions of the sections. However, the total of measured acceleration time series of the study sites can clearly be distinguished from each other.

Gas exchange velocity and drifter acceleration are both driven by the surface roughness, i.e. by the near surface flow turbulence and waves. Consequently, a linear relationship between $k_{600}$ and $S D_{\mathrm{a}}$ was observed for $k_{600}>7 \mathrm{~m} \mathrm{~d}^{-1}$ (Fig.2b).
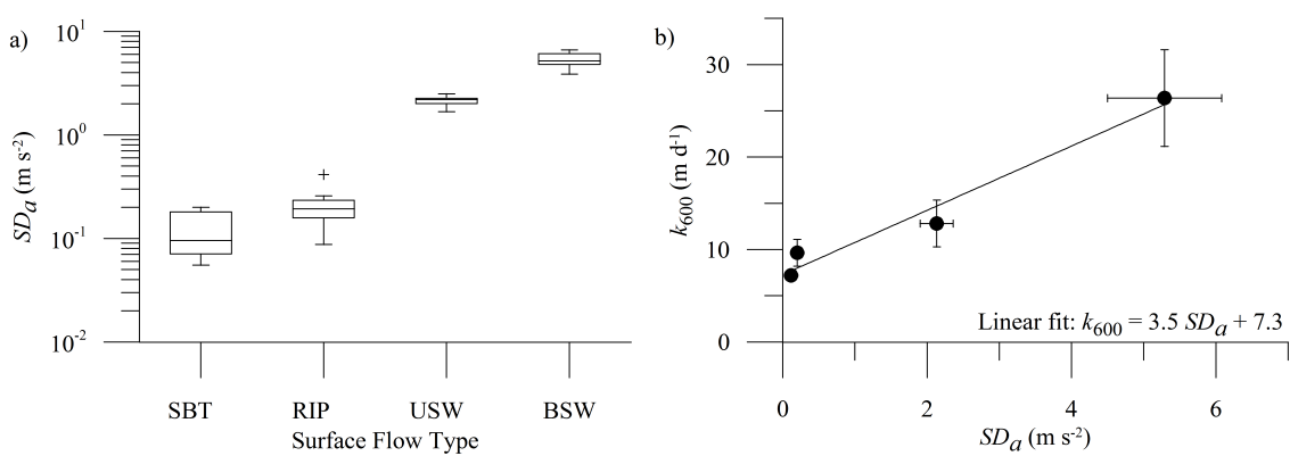

Fig. 2. a) Box plot of the standard deviations of instantaneous drifter acceleration $\left(S D_{a}\right)$ estimated for individual runs at stream sections with different surface flow type (SBT, RIP, USW and BSW). The boxes encompass the $25-75$ percentile range, whiskers show minimum and maximum, and horizontal lines mark median values. The outlier (factor: 1.5) at RIP is denoted by the cross. b) Gas exchange velocity $k_{600}$ (based on $\mathrm{CO}_{2}$ ) versus standard deviation of drifter acceleration. Horizontal and vertical error bars show standard deviations of different drifter runs and chamber deployments, respectively.

\subsection{Flow and Turbulence}

Measurement results on both section- (Table 1) and spot-scale (Table 2) show clear differences between SFT. Although all flows were subcritical $(\mathrm{Fr}<1)$, parameters increased - starting from the smoothest SBT, followed by RIP and USW - with the surface flow types. The largest difference between the SFT appears for the water surface slope of the USW in comparison to that of RIP and SBT (factors of 27 and 53, respectively). Also flow velocity, irrespective of mean section-scale or spot-scale velocities, as well as the turbulent kinetic energy and Reynolds-number were highest at USW, followed by RIP and SBT. Dissipation rates of turbulent kinetic energy (Fig.3a) varied i) with the surface flow types (two to three orders of magnitude, largest dissipation rates at USW), ii) with the applied method in case of section scale estimates $\left(\varepsilon_{D} / \varepsilon_{S} \approx 10\right)$, and iii) at USW spots between velocity components $\left(\varepsilon_{u} / \varepsilon_{w} \approx 10\right)$. The vertical profiles of dissipation rates of vertical velocity fluctuations were rather constant (except one spot at USW) and mean dissipation rates scaled with spot mean velocities $\left(\varepsilon_{w} \sim U^{3}\right)$.

Further insights in the flow and turbulence structure below the air-water interface were detectable in the space- and time-resolved velocity vectors and in the spectra of turbulent velocity fluctuations (not shown). Briefly, flow patterns obtained at SBT and RIP did not differ notably. The patterns consisted of 5 to $10 \mathrm{~cm}$ sized turbulent eddies of random direction of rotation. These were predominantly advected horizontally. Turbulent eddies at USW were less persistent, i.e. generated and dissipated rapidly over short distances. 
Analysis of the power spectral density of vertical velocity fluctuations (at USW also of the longitudinal component) revealed dominant peaks at wavelengths corresponding to the scale of the water depth $\left(\kappa_{H}\right)$. In case of RIP and SBT, peaks in the spectra of longitudinal velocity fluctuations appear at wavenumbers corresponding to the scale of the stream widths. The inertial subranges in the spectra of the longitudinal and vertical components at SBT and RIP (at wavelengths $\kappa>\kappa_{H}$ ) were almost identical. At USW, power spectral density of the longitudinal component was much higher than that of the vertical, which denotes strong anisotropy of the turbulence even in the inertial subrange.

Overall, gas exchange velocities increased with dissipation rates to the power of $1 / 4$ (Fig.3b). This relationship appeared stronger for dissipation rates obtained from vertical velocities (spots) and from the shear stress approach (sections) in comparison to that obtained from the longitudinal components and from the drag based approach, respectively. The (mean) scaling coefficient between the gas exchange velocity and the normalized dissipation rate was $\alpha=0.5$, while the drag-based approach provided the lowest $\left(\alpha\left(\varepsilon_{D}\right)=\right.$ $0.37)$ and the shear stress approach the highest $\left(\alpha\left(\varepsilon_{S}\right)=0.63\right)$ mean value of the scaling coefficient.
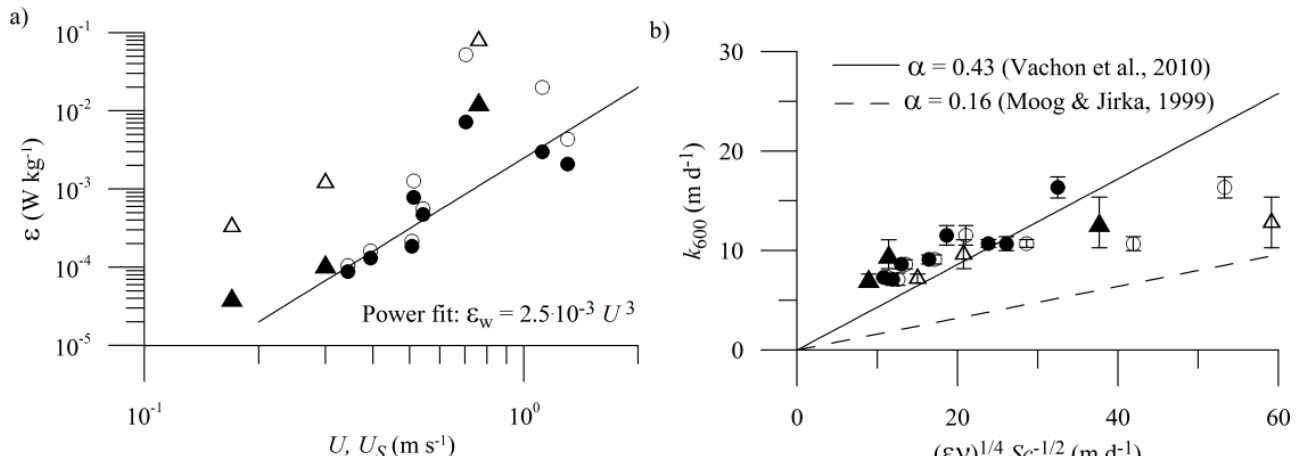

Fig. 3. a) Dissipation rates of turbulent kinetic energy versus mean velocity. Open circles denote dissipation rates of longitudinal velocity fluctuations $\left(\varepsilon_{\mathrm{u}}\right)$ and closed circles of vertical velocity fluctuations $\left(\varepsilon_{\mathrm{w}}\right)$ over spot mean flow velocity. Triangles denote section-scale dissipation rates of turbulence (open: drag-based approach $\left(\varepsilon_{\mathrm{D}}\right)$, closed: shear stress approach $\left(\varepsilon_{\mathrm{S}}\right)$ ) over mean near surface velocity. b) Gas exchange velocity $\left(k_{600}\right)$ versus its universal scaling function (Eq.1), where symbols of the dissipation rates correspond to that of Fig.3a). Lines show scaling factors obtained in two other studies.

\section{Discussion}

We showed that the dependency of $k_{600}$ on dissipation rates followed the small eddy-model (Eq.1), most appropriate with a scaling coefficient derived from measurements in a winddriven system [5]. The scaling coefficient obtained from open channel experiments [1], however, underestimated measured $k_{600}$ at the investigated stream. Isotopic conditions in the smooth flow sections (SBT and RIP) provided similar dissipation rates estimated from both longitudinal and vertical velocity components, but strong anisotropy at the rough flow required using dissipation rates from vertical components to estimate gas exchange velocities. Technical limitations of the PIV impeded highly resolved turbulence measurements directly at the air-water interface. However, although dissipation rates likely decrease towards and in vicinity to the air-water interface, vertical profiles of $\varepsilon_{\mathrm{w}}$ were rather constant, i.e. independent of the vertical position of the measurement. Comparable dissipation rates were obtained from bulk parameters, particularly on the basis of the shear stress approach, which indicates smooth flow conditions with small roughness, and small 
eddy driven gas exchange velocities. The drag-based approach, in contrast, provided much higher values.

The close relationship between the standard deviation of the drifter accelerations and the gas exchange velocity provides first evidence that water surface roughness is caused by the near-surface hydraulic conditions, which are also relevant for the gas exchange across the air-water interface. Furthermore, the surface roughness measured by the drifter differed strongly between different surface flow types (and corresponding bulk hydrometrics). Hence, drifter measurements and surface flow type mapping (frequently part of comprehensive monitoring programs) might be promising approaches for improved reachand catchment-scale estimations of gas exchange velocities. However, further investigations are necessary to cover a wider range of stream types, flow and turbulence conditions, and gas exchange velocities. The range of $k_{600}$ values in this study was small in comparison to others, which show exchange coefficients varying by two orders of magnitude at the catchment scale [6, 7]. However, in comparison to these studies, which cover 4 to 5 different stream orders, our study illustrates the large spatial variability of $k_{600}$ even within one stream order and one stream type.

We thank Christoph Bors for his help in preparing and conducting the measurements. This study was financially supported by the German Research Foundation (DFG, grant LO1150/9-1) and the Stiftung Rheinland-Pfalz für Innovation (Project 961-386261/1054).

\section{References}

1. D. Moog and G. Jirka, JHE, 125, 11-16 (1999)

2. P.A. Raymond, C.J. Zappa, D. Butman, T.L. Bott, J. Potter, et al., L\&O: F\&E, 2, 41-53 (2012)

3. B. Jähne and H. Haußecker, Ann. Rev. of Fluid Mechanics, 30, 443-468 (1998)

4. G. Katul and H. Liu, GRL, 44, 1892-1898 (2017)

5. D. Vachon, Y.T. Prairie and J.J. Cole, L\&O, 55, 1723-1732 (2010)

6. J. Schelker, G.A. Singer, A.J. Ulseth, S. Hengsberger and T.J. Battin, L\&O, 61, 18261838 (2016)

7. M.B. Wallin, M.G. Öquist, I. Buffam, M.F. Billett, J. Nisell, et al., Global Biogeochem. Cy., 25, n/a-n/a (2011)

8. M.D. Newson and C.L. Newson, Prog. Phys. Geog., 24, 195-217 (2000)

9. V. Dahm, B. Kupilas, P. Rolauffs, D. Hering, P. Haase, et al. (Umweltbundesamt 2014)

10. A. Lorke, P. Bodmer, C. Noss, Z. Alshboul, M. Koschorreck, et al., Biogeosciences, 12, 7013-7024 (2015)

11. Z. Alshboul, J. Encinas-Fernández, H. Hofmann and A. Lorke, ES\&T, 50, 5555-5563 (2016)

12. J.A. Goldenfum (UNESCO/IHA 2010)

13. W.J. Emery and R.E. Thomson, Data analysis methods in physical oceanography (Elsevier, 2004)

14. D.G. Goring and V.I. Nikora, JHE, 128, 117-126 (2002)

15. C.E. Bluteau, N.L. Jones and G.N. Ivey, L\&O: Methods., 9, 302-321 (2011) 